\title{
HARMONIC MEASURE VERSUS HAUSDORFF MEASURES ON REPELLERS FOR HOLOMORPHIC MAPS
}

\author{
ANNA ZDUNIK
}

\begin{abstract}
This paper is a continuation of a joint paper of the author with $F$. Przytycki and M. Urbański. We study a harmonic measure on a boundary of so-called repelling boundary domain; an important example is a basin of a sink for a rational map. Using the results of the above-mentioned paper we prove that either the boundary of the domain is an analytically embedded circle or interval, or else the harmonic measure is singular with respect to the Hausdorff measure corresponding to the function $\phi_{c}(t)=t \exp \left(c \sqrt{\log \frac{1}{t} \log \log \log \frac{1}{t}}\right)$ for some $c>0$.
\end{abstract}

\section{INTRODUCTION AND STATEMENT OF RESULTS}

Let $\Omega$ be a simply connected domain in the Riemann sphere $\overline{\mathbb{C}}$, $\operatorname{card}(\overline{\mathbb{C}}-\Omega)>2$. Let $R: D \rightarrow \Omega$ be the Riemann map from the unit disc $D$ onto $\Omega$. Since $R$ has nontangential limits almost everywhere on $S^{1}=\partial D$ [D], the image $R_{*} l$ of the length measure $l$ on $S^{1}$ can be considered.

This measure (denoted usually by $\omega$ and called a harmonic measure on $\partial \Omega$ ) has been studied a long time (see [Mk2 and PUZ] for the corresponding references), with the most interesting results obtained recently in two remarkable papers, [Mk1 and $\mathrm{Mk} 2]$.

For an increasing function $\phi: \mathbb{R}_{+} \rightarrow \mathbb{R}_{+}, \phi(0)=0$ we define an outer measure for $A \subset \overline{\mathbb{C}}$ :

$$
\Lambda_{\phi}(A)=\lim _{\delta \rightarrow 0} \inf \left\{\sum_{j} \phi\left(\operatorname{diam} B_{j}\right)\right\},
$$

where the infimum is taken over all coverings of $A$ with balls of a diameter smaller than $\delta$ (the diameter is computed with respect to the spherical metrics on $\overline{\mathbb{C}}$ ). In particular, for $\phi(t)=t^{\alpha}$, we obtain the $\alpha$-dimensional Hausdorff measure. $\Lambda_{t^{\circ}}$ will be denoted by $\Lambda_{\alpha}$.

Let $\mu$ be a probability measure on Borel subsets of $\overline{\mathbb{C}}$. Hausdorff dimension of $\mu$ is defined as

$$
\operatorname{HD}(\mu)=\inf _{E: \mu(E)=1} \operatorname{HD}(E) .
$$

Received by the editors July $18,1989$.

1980 Mathematics Subject Classification (1985 Revision). Primary 58F11. 
We say that $\mu$ is singular with respect to $\Lambda_{\phi}$, if for every Borel set $E \subset \overline{\mathbb{C}}$, $\Lambda_{\phi}(E)=0 \Rightarrow \mu(E)=0 ; \mu$ is singular with respect to $\Lambda_{\phi}$ if there exists a Borel set $E \subset \overline{\mathbb{C}}$ such that $\mu(E)=1$ and $\Lambda_{\phi}(E)=0$. Notice that $\mu \perp \Lambda_{\alpha}$ implies that $\operatorname{HD}(\mu) \leq \alpha$, while $\mu \ll \Lambda_{\alpha}$ implies that $\operatorname{HD}(\mu) \geq \alpha$.

In his remarkable paper [Mk1], N. G. Makarov proved that

$$
\omega \perp \Lambda_{\alpha} \text { for all } \alpha>1,
$$

solving the conjecture due to Øksendal [Ø].

In [Mk2], Makarov gave a proof of the equality $\operatorname{HD}(\omega)=1$ for every Jordan domain (this result was generalized afterwards for an arbitrary simply connected domain).

Moreover, he obtained the following "universal estimate" of $\omega$ : there exists a constant $C>0$ (independent of a domain) such that

$$
\omega \ll \Lambda_{\phi_{C}},
$$

where $\Lambda_{\phi_{C}}$ is a Hausdorff measure corresponding to the function

$$
\phi_{C}(t)=t \exp \left(C \sqrt{\log \frac{1}{t} \log \log \frac{1}{t}}\right) .
$$

Now, (2) immediately leads to

$$
\omega \ll \Lambda_{\alpha} \text { for all } \alpha<1 .
$$

Putting together (1) and (3), one obtains

$$
\mathrm{HD}(\omega)=1 .
$$

We are going to study the harmonic measure on the boundary of a domain on which some special holomorphic dynamics exist. Below we give a definition.

Definition. Let $\Omega$ be a simply connected domain with $\operatorname{card}(\overline{\mathbb{C}}-\Omega)>2$. Assume there exists a holomorphic map $f$ defined on a neighborhood $U$ of $\partial \Omega$ such that

$$
f(U \cap \Omega) \subset \Omega, \quad f(\partial \Omega)=\partial \Omega,
$$

and

$$
\bigcap_{n=1}^{\infty} f^{-n}(U \cap \Omega)=\partial \Omega .
$$

Then $\Omega$ is called a repelling boundary domain (RB-domain).

The RB-domains were studied in [P1, P2 and PUZ]. The most important example of such a domain (which motivated the definition above) is a basin of a sink for a rational map.

For RB-domains there exists an invariant, ergodic measure on $\partial \Omega$, equivalent with $\omega$ (see $\S 1$ ), and the proof of the equality $\operatorname{HD}(\omega)=1$ can be obtained by use of dynamical methods. A. Manning was the first to give the proof for 
a basin of attraction of $\infty$ for a polynomial with expanding property on the Julia set.

A general proof (for an arbitrary RB-domain) was given in [P1 and P2].

In [PUZ] we studied the relation between $\omega$ and various Hausdorff measures, showing that usually $\omega$ happens to be "very singular." More precisely, a striking dichotomy was observed:

Theorem A (see [PUZ, §6]). Assume that $\Omega$ is an RB-domain. Then there exists $c(\omega) \geq 0$ such that

$$
\begin{aligned}
& \omega \perp \Lambda_{\phi_{c}} \text { for every } c \in(0, c(\omega)), \\
& \omega \ll \Lambda_{\phi_{c}} \text { for every } c>c(\omega) .
\end{aligned}
$$

$c(\omega)$ is called a transition parameter.

Obviously, only $c(\omega)$ strictly positive gives us the required singularity of $\omega$.

In [PUZ] we were only in a position to check that in the case of $f_{\mid \partial \Omega}$ expanding either $c(\omega)$ is positive or $\partial \Omega$ is a real-analytic Jordan curve.

The aim of the present paper is to study a general (nonexpanding) case. We prove the following result:

Theorem. If $\Omega$ is an RB-domain, then the transition parameter $c(\omega)$ is always positive (and thus $\omega$ is as singular as in Theorem A of [PUZ]) except for two cases: $\partial \Omega$ is a real-analytic Jordan curve or an analytically embedded closed interval.

This theorem was stated as a conjecture in [PUZ]. The other conjecture was that $\operatorname{HD}(\partial \Omega)=1$ also implies that $\partial \Omega$ is an analytic Jordan curve or an analytic interval. This conjecture can be proved by adapting the methods of $[\mathrm{Z}]$.

\section{Steps OF PROOF}

First, recall some notations and facts proved in [P1, P2, and PUZ]. Let $R: D \rightarrow \Omega$ be the Riemann map. Let $g=R^{-1} \circ f \circ R$. The map $g$ is defined on some annulus $P=\left\{z \in \mathbb{C}: r_{0}<|z|<1\right\}$. The map $g$ extends to the closed annulus cl $P$, leaves the unit circle $S^{1}$ invariant and therefore it can be extended beyond $\operatorname{cl} D$ (by the symmetry principle). Moreover $g_{\mid S^{1}}$ is expanding (see $[\mathrm{P} 2, \S 7]$ for the proofs of these facts).

There exists a measure $\nu$ on $S^{1}$, equivalent to the Lebesgue measure, invariant under $g$ (a Gibbs measure for $-\log \left|g^{\prime}\right|$ ). The measure $\mu=R_{*} \nu$ is well defined (since $R$ has nontangential limits $\nu$-a.e.) and equivalent to $\omega$.

Let $\varphi=\log \left|\frac{f^{\prime} \circ R}{g^{\prime}}\right|$. Then $\varphi \in L^{2}\left(S^{1}, \nu\right)$ (see [PUZ, $\S 5$, Lemma 4]) and $\int \varphi d \nu=0[\mathrm{P} 2$, Theorem 2].

We study a behavior of the sequence of random variables

$$
n \rightarrow S_{n} \varphi=\varphi+\varphi \circ g+\cdots+\varphi \circ g^{n-1} \text {. }
$$


This sequence indicates how strong the boundary compression is under $R$. (To see this, assume for a moment that $F_{\mid \partial \Omega}$ is expanding (then $R$ extends continuously to $\operatorname{cl} D$ ). Let $B$ be a small ball around $p \in S^{1}$; take the first $n$ such that $g^{n} B$ is large. Then $\frac{1}{\left|\left(g^{n}\right)^{\prime}(p)\right|}$ is (up to a constant factor) a diameter of $B$, while $\frac{1}{\left|\left(f^{n}\right)^{\prime}(R(p))\right|}$ is (up to a constant factor) a diameter of $R(B)$. Thus, $\exp S_{n} \varphi$ equals (up to a bounded factor) $\frac{\operatorname{diam} B}{\operatorname{diam} R(B)}$. The same idea applies also in a general, nonexpanding case.)

Let $\mathscr{A}$ be a Markov partition for $g$. By $\mathscr{A}^{n}$ we denote a partition $\mathscr{A} \vee$ $g^{-1} \mathscr{A} \vee \cdots \vee g^{-(n-1)} \mathscr{A}$.

The asymptotic variance for the sequence $S_{n} \varphi$ is defined by

$$
\sigma^{2}=\lim _{n \rightarrow \infty} \frac{\int\left(S_{n} \varphi\right)^{2} d \nu}{n} .
$$

This limit exists since we have

$$
\int\left|\varphi \cdot\left(\varphi \circ g^{n}\right) d \nu\right| \leq K \beta^{n}
$$

for some $K>0, \beta \in(0,1)$, which in turn follows (as in [Bo, 1.26]) from

$$
\left|\nu\left(g^{-n}(A) \cap B\right)-\nu(A) \nu(B)\right| \leq \nu(A) \nu(B) \beta^{n-s},
$$

where $A, B$ are cylinders, $s=$ length of $A$ [Bo, 1.14], and

$$
\int\left|\varphi-E\left(\varphi \mid \mathscr{A}^{n}\right)\right|^{2+\delta} d \nu \leq K \beta^{n} \text { for some } \delta>0, K, \beta \text { as above, }
$$

(a quick approximation by conditional expectations with respect to $\mathscr{A}^{n}$, see [PUZ, §5, Lemma 5]).

If $\sigma^{2} \neq 0$, then the properties (3), (4), and (5) allow us to conclude that the sequence $S_{n} \varphi$ satisfies the Law of Iterated Logarithm (it can be deduced from a general Theorem 7.1 in [Ph-St], see also [PUZ, $\S 1,(14)]$ :

$$
\limsup _{n \rightarrow \infty}\left(\sum_{j=0}^{n-1} \varphi \circ g^{j}\right) / \sqrt{n \log \log n} \stackrel{\text { a.e. }}{=} \sqrt{2 \sigma^{2}} .
$$

Property (6) leads (directly, but through many essential difficulties) to the required singularity of $\omega$ with transition parameter

$$
c(\omega)=\sqrt{\frac{2 \sigma^{2}}{\lambda}},
$$

where $\lambda=\int \log \left|f^{\prime}\right| d R_{*} \nu$ is a Lyapunov exponent.

Our purpose is to check that $\sigma^{2} \neq 0$ if $\partial \Omega$ is neither an analytic Jordan curve nor an analytic closed interval.

We start the considerations with a well-known lemma ([PUZ, §1, Lemma 1]). 
Lemma. Under the assumptions above, the asymptotic variance $\sigma^{2}$ is zero iff $\varphi$ is homologous to zero in $L^{2}(\nu)$, i.e.

$$
\log \left|f^{\prime} \circ R\right|-\log \left|g^{\prime}\right|=u \circ g-u \quad \text { for some } u \in L^{2}(\nu) \text {. }
$$

Our first step is to show that if $\sigma^{2}=0$, then the derivative $R^{\prime}$ of the Riemann map has nontangential limits in $l$-almost all points in $S^{1}$. The logarithm of the limit function belongs to $L^{2}(\nu)$ and can be taken as $u$ in the formula (8). This is done in $\S 2$.

The next essential step is to prove that the Riemann map extends continuously to $\operatorname{cl} D$. Moreover, $R^{\prime}$ extends to a continuous function defined on $\operatorname{cl} D$. This will be proved in $\S 3$.

The method is close to that used in [Z] and exploits an explicit construction of the Pesin set, which has been done in [PUZ, §5, Lemma 8].

Next, we study the possible behavior of trajectories of critical points in $\partial \Omega$. We show that each critical trajectory must fall into a source (Proposition $4, \S 4$ ).

In Proposition 5, $\S 5$, one shows that in the neighborhood of a point $p$ which is not a critical value, $\partial \Omega$ is a smooth arc. This leads easily to the conclusion that $\partial \Omega$ is a smooth Jordan curve or a smoothly embedded closed interval (Corollary $4, \S 5$ ).

We complete the proof by showing that $\partial \Omega$ is actually analytic. To prove this, we adapt the Sullivan's argument [Su; P3; PUZ, §6, Step 5 of the proof of Theorem C].

We start with a proposition which has been already stated in [PUZ] under an additional assumption that $f$ is defined on the neighborhood of the whole $\Omega$. The proof which we give here allows us to omit this assumption.

$R^{\prime}$ will denote the usual derivative, $f^{\prime}$ is (in general) the spherical derivative.

Proposition 1. If $\Omega$ is an $R B$-domain and $\log \left|f^{\prime} \circ R\right|$ is homologous to $\log \left|g^{\prime}\right|$ in $L^{2}\left(S^{1}, \nu\right)$, then the function $R^{\prime}$ has nontangential limits almost everywhere.

Proof. First, suppose that $\operatorname{cl} \Omega \neq \overline{\mathbb{C}}$, then one can assume that $\infty \notin \operatorname{cl} \Omega$ and one can consider the usual derivative $f^{\prime}$ instead of the spherical one. Denote

$$
\varphi=\log \left|f^{\prime} \circ R\right|-\log \left|g^{\prime}\right| \text {. }
$$

By the assumption, we have

$$
\varphi=u \circ g-u
$$

for some $u \in L^{2}\left(S^{1}, \nu\right)$.

For a function $h \in L^{2}\left(S^{1}, \lambda\right)$, we denote by $\bar{h}$ the extension of $h$ given by the Poisson integral. Notice that $\bar{h}$ belongs to the Hardy space $h^{2}$ and has a nontangential limit equal to $h$ almost everywhere [D]. 
Let $\bar{\varphi}$ be an extension of $\varphi$ obtained as above and let

$$
v=\bar{\varphi}-\log \left|\frac{f^{\prime} \circ R}{g^{\prime}}\right| ;
$$

$v$ is defined on some annulus $P=\left\{z \in \mathbb{C}: r_{0}<|z|<1\right\}$. (Notice that $v=0$ if $f$ extends to the whole $\Omega$.)

Denote by $w$ the difference

$$
w=\overline{u \circ g}-\bar{u} \circ g .
$$

(Again, $w=0$ if $f$ is defined on the whole $\Omega$, since $\bar{u} \circ g$ is harmonic in this case.)

Since $\varphi=u \circ g-u$ a.e. in $S^{1}$, it follows that

$$
\bar{\varphi}=\overline{u \circ g-u}=\overline{u \circ g}-\bar{u}=\bar{u} \circ g-\bar{u}+w .
$$

Thus,

$$
\log \left|\frac{f^{\prime} \circ R}{g^{\prime}}\right|=\bar{\varphi}-v=\bar{u} \circ g-\bar{u}+w-v \quad \text { in } P .
$$

For $x \in P$ such that $x, g(x), \ldots, g^{n}(x) \in P$, we have

$$
\log \left|\frac{\left(f^{n}\right)^{\prime}(R(x))}{\left(g^{n}\right)^{\prime}(x)}\right|=\bar{u}\left(g^{n}(x)\right)-\bar{u}(x)+S_{n} w(x)-S_{n} v(x),
$$

where $S_{n} w(x)=\sum_{i=0}^{n-1} w\left(g^{i}(x)\right)$, analogously $S_{n} v(x)$.

We need a simple

Lemma 1. Both $v$ and $w$ extend to Lipschitz-continuous functions $\operatorname{cl} P, v_{\mid S^{1}}=$ $w_{\mid S^{1}}=0$.

Assume that Lemma 1 is proved.

Let $x \in P$; take the smallest $n$ such that $g^{n}(x) \notin P$. By (7) we have

$$
\begin{aligned}
& -\log \left|R^{\prime}(x)\right|+\log \left|R^{\prime}\left(g^{n}(x)\right)\right|=\log \left|\frac{\left(f^{n}\right)^{\prime}(R(x))}{\left(g^{n}\right)^{\prime}(x)}\right| \\
& =\bar{u} \circ g^{n}-\bar{u}-S_{n} v(x)+S_{n} w(x) .
\end{aligned}
$$

Since $v$ and $w$ are Lipschitz-continuous, $v_{\mid S^{1}}=w_{\mid S^{1}}=0$, and $g$ is expanding, both sums $S_{n} v$ and $S_{n} w$ can be estimated by

$$
C \cdot \sum_{i=0}^{n-1} \operatorname{dist}\left(g^{i}(x), S^{1}\right)<D<\infty,
$$

where $C, D$ are some constants. Also, $\log \left|R^{\prime}\left(g^{n}(x)\right)\right|$ and $\bar{u}\left(g^{n}(x)\right)$ are bounded since $g^{n}(x)$ is far from $S^{1}$. Thus, using (8), we conclude that the difference $\log \left|R^{\prime}\right|-\bar{u}$ is bounded. Thus, the integrals $\int_{r S^{1}}\left(\log \left|R^{\prime}\right|\right)^{2}$ are bounded 
because this is the case for the integrals $\int_{r S^{1}} \bar{u}^{2}$ ( $\bar{u}$ belongs to the Hardy space $\left.h^{2}\right)$.

It follows that $R^{\prime}$ is in the Nevanlinna class (i.e. $\int_{r S^{1}} \log _{+}\left|R^{\prime}\right|$ are bounded) and therefore $R^{\prime}$ has nontangential limits almost everywhere [D].

It remains to consider the case $\operatorname{cl} \Omega=\overline{\mathbb{C}}$. Then one can assume that $\infty \in$ int $\Omega$. Take a univalent representation $\mathscr{R}: D_{\infty}=\{z:|z|>1\} \rightarrow \Omega$ such that $\infty \rightarrow \infty$.

We repeat the proof above with the functions $v, w$, replaced by $v_{1}, w_{1}, \varphi_{1}$ where $v_{1}(z)=v\left(\frac{1}{z}\right)$, similarly for $w_{1}$ and $\varphi_{1}$. The proof of Lemma 1 changes slightly. The conclusion is that the function

$$
D \ni z \mapsto \mathscr{R}^{\prime}\left(\frac{1}{z}\right) \text { has nontangential limits a.e. }
$$

Since $R$ can be taken as $\mathscr{R}\left(\frac{1}{z}\right)$, also $R^{\prime}$ has nontangential limits a.e.

Proof of Lemma 1. First, notice that both $v$ and $w$ have nontangential limits equal to zero a.e. Indeed, recall that $v=\bar{\varphi}-\log \left|\frac{f^{\prime} \circ R}{g^{\prime}}\right|, w=\overline{u \circ g}-\bar{u} \circ g$. Both $\bar{\varphi}$ and $\log \left|\frac{f^{\prime} \circ R}{g^{\prime}}\right|$ have nontangential limits equal to $u \circ g-u$ a.e.

We shall show that also the integrals $\int_{r S^{1}}|v|$, and $\int_{r S^{1}}|w|$ tend to zero as $r \rightarrow 1$, by finding integrable majorants.

Let $x \in S^{1}$. We have

$$
|v(r x)| \leq|\bar{\varphi}(r x)|+|\log | \frac{f^{\prime} \circ R}{g^{\prime}}|(r x)| .
$$

Now, $\bar{\varphi}(r x)$ can be estimated (independently by $r$ ) by the function $x \mapsto$ $\sup _{0 \leq \rho<1}|\bar{\varphi}(\rho x)|$ (integrable by the Hardy-Littlewood maximal theorem, see [D]).

Next, notice that

$$
|\log | f^{\prime} \circ R|(z)| \leq C \cdot \sum_{c_{i}} \log \left|R(z)-c_{i}\right|
$$

for $z \in P$, where $C$ is some constant and $c_{i}$ are critical points of $f$ in $\operatorname{cl} R(P)$.

But $\log \left|R(z)-c_{i}\right|$ belongs to the Hardy space $h^{p}$ for all $p>0$. Indeed, fix $p>0$ and $\alpha \in\left(0, \frac{1}{2}\right)$, then

$$
|\log | R(z)-c_{i}||^{p} \leq D \cdot \frac{1}{\left|R(z)-c_{i}\right|^{\alpha}}
$$

where $D$ is some positive constant. (Recall that $R$ is bounded.) The functions $z \mapsto \frac{1}{R(z)-c_{i}}$ are univalent. Thus, the integrals $\int_{r S^{1}} \frac{1}{\left|R-c_{i}\right|^{10}}$ are bounded [D].

Hence, the function

$$
H(x)=\sum_{i} \sup _{0 \leq r<1}|\log | R-c_{i}||^{p}
$$

(being a majorant of $\log \left|f^{\prime} \circ R\right|(r z)$ ) is integrable, again by the Hardy-Littlewood maximal theorem. 
The conclusion is that $\int_{r S^{1}}|v| \rightarrow 0$ as $r \rightarrow 1$.

Now, we check the same for $w$. Recall that $\int_{r S^{1}} w=\int_{r S^{1}} \bar{u} \circ g-\int_{r S^{1}} \bar{u} \circ g$. We have

$$
\int_{r S^{1}} \overline{u \circ g} \underset{r \rightarrow 1}{\longrightarrow} \int_{S^{1}} u \circ g
$$

since $\overline{\bar{u} \sigma} \in h_{2}$.

The second integral equals $r \cdot \int_{\vartheta \in(0,2 \pi)} \bar{u} g\left(r e^{i \vartheta}\right) d \vartheta$. Since the argument of $g\left(r e^{i \vartheta}\right)$ changes monotonically with $\vartheta$ (for $r$ close to 1 ), this can be estimated by $D \cdot \int_{S^{1}} \sup _{0 \leq \rho<1} \bar{u}(\rho x)$ ( $D$ is some constant). The integral above is finite by the Hardy-Littlewood maximal theorem.

Thus, also $\int_{r S^{1}}|w| \rightarrow 0$ as $r \rightarrow 1$. The proof of Lemma 1 can now be finished with the following (rather standard) argument:

Let $V$ be the unique function harmonic in $P$, continuous in $\bar{P}$ with boundary functions: zero on $S^{1}$ and $v$ on $r_{0} S^{1}$. (Recall that $P=\left\{z: r_{0}<|z|<1\right\}$.) We have

$$
V(x)=\int_{P\left(R, r_{0}\right)} \frac{\partial g_{R}}{\partial n}(y, x) V(y) d l(y)
$$

for $x \in P\left(R, r_{0}\right)=\left\{z: r_{0}<|z|<R<1\right\}$, where $g_{R}$ is the Green's function for $P_{R, r_{0}}$. The same is true for $v$ and, consequently, for $V-v$.

When $R \rightarrow 1$, the integral $\int_{P\left(R, r_{0}\right)} \frac{\partial g_{R}}{\partial n}(y, x)(V-v)(y) d l(y)$ tends to zero since $\frac{\partial g_{R}}{\partial n}$ are uniformly bounded and $\int_{P\left(R, r_{0}\right)}|V-v|$ tend to zero. Hence, $V-v \equiv 0$.

The conclusion is that $v$ is continuous in $\mathrm{cl} P, v_{\mid S^{1}}=0$. Now, $v$ can be extended to a harmonic function in some annulus $P_{r_{0},(1+\varepsilon)}$ (see [Go, Chapter VI, §3]). Thus, $v$ is Lipschitz-continuous in $\operatorname{cl} P$.

The same argument works for $w$.

\section{3. $\partial \Omega$ IS SMOOTH}

Proposition 1 allows us to deduce the crucial

Theorem 1. If $\Omega$ is an RB-domain and the derivative of the Riemann map $R: D \rightarrow \Omega$ has nontangential limits a.e., then $R$ extends to a continuous map on the closed disc $\bar{D}$ and the derivative (spherical) is bounded in $D$.

Before proving Theorem 1 we introduce some notations.

For $x \in \bar{D} \backslash\{0\}$, let $r_{x}$ denote the ray containing $x \quad\left(r_{x}=\{t \cdot x: 0 \leq t \leq\right.$ $\left.\left.\frac{1}{|x|}\right\}\right)$. Take $\alpha \in(0, \pi)$ and $x \in D, x \neq 0$. Let $C(x)$ be a cone bounded by two straight lines intersecting at $x$, such that the angle between each line and $r_{x}$ equals $\alpha / 2 . C$ is a sum $C_{1} \cup C_{2}$ of interiors of two angles. We denote by $T(x)$ the set $C_{i} \cap D$, where $C_{i}$ is chosen so that $0 \notin C_{i}$ (Figure 1). Similarly, for $y \in S^{1}$ by $S(y)$ we denote $C_{j} \cap D$, where $C_{j}$ is chosen so that $0 \in C_{j}$ (Figure 2). 


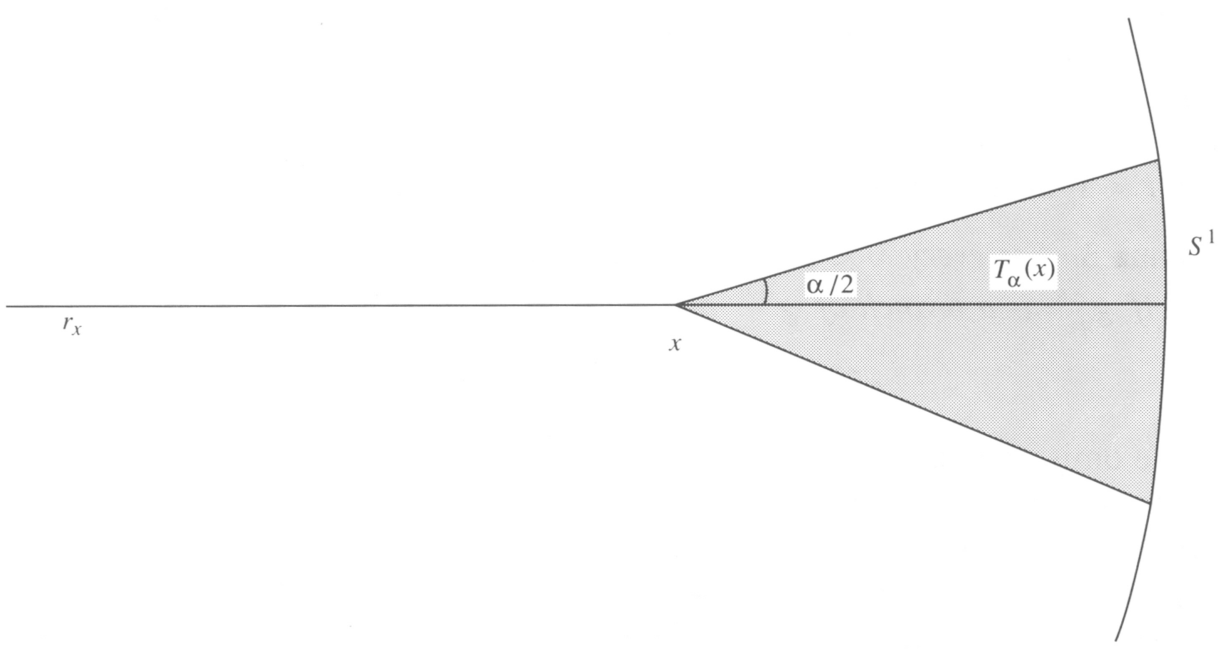

Figure 1

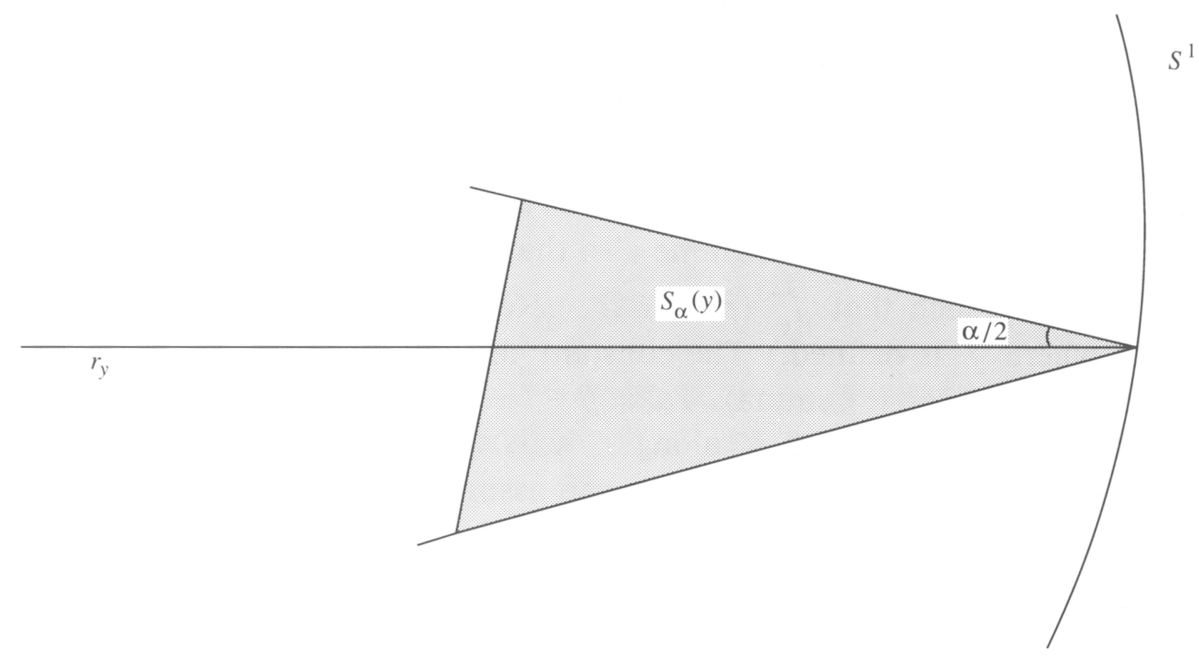

FIGURE 2

Fix $1>\rho_{0}>0$ so that $g$ is expanding in the annulus $P_{\rho_{0}}=\left\{z \in \mathbb{C}: \rho_{0}<\right.$ $|z|<1\}$.

One can assume that $\infty \notin \partial \Omega$ and $\infty \notin R(P) . R^{\prime}$ will denote the usual derivative.

We shall prove that $R_{\mid P_{\rho_{0}}}^{\prime}$ is bounded.

We need some technical lemmas.

Lemma 2 (see [P2, §2]). There exists $C>0$ such that for every simply connected domain $V \subset P$ such that diam $V<\delta<1-\rho_{0}$ and for every branch $g^{-n}$ defined 
on $V$

$$
\sup _{z, w \in V}\left|\left(g^{-n}\right)^{\prime}(z) /\left(g^{-n}\right)^{\prime}(w)-1\right|<C \cdot \delta .
$$

For $r<1$ denote by $P_{r, 1}$ the set $\{z \in \mathbb{C}: r<|z|<1\}$.

Lemma 3. For every $0<\alpha<\pi$ and $\beta \in(\alpha, \pi)$ there exists $r \in\left(\rho_{0}, 1\right)$ such that if $g_{\eta}^{-n}$ is a branch of $g^{-n}$ defined in $S_{\alpha}(y) \cap P_{r, 1}\left(y \in S^{1}\right)$, then

$$
g^{-n}\left(S_{\alpha}(y) \cap P_{r, 1}\right) \subset S_{\beta}\left(g_{\eta}^{-n}(y)\right) \text {. }
$$

We omit easy proofs.

We say that $q$ is a critical value if $q=f^{n}(c)$ for some $n \geq 1$ and a critical point $c \in \partial \Omega$ (critical trajectories coming from beyond $\mathrm{cl} \Omega$ are unsubstantial).

First, choose a point $\tau \in S^{1}$ such that $R$ has the radial limit $p$ at $\tau$. We assume that $p$ is not a critical value of $f$ (this can be done, since $\omega$ (critical values of $f(=0$ ). The proof of Theorem 1 is based on Propositions 2 and 3 below.

Proposition 2. (a) There exists a disc $B$ around $p$ such that for every $\alpha \in(0, \pi)$ there exist $1>r_{1}>0$ such that if $x \in r_{\tau}$ and $|x|>r_{1}$, then $R\left(T_{\alpha}(x)\right) \subset B$.

(b) The derivative $R^{\prime}$ is bounded in $T_{\alpha}(x)$.

Proof. Let $B$ be a disc around $p$ such that there are no critical values up to order $M$ in $2 \cdot B$ (where by $2 \cdot B$ we denote a disc with the same center and with a radius twice as large). We assume also that $f_{\eta}^{-i}(2 \cdot B) \subset U$ for every branch $f_{\eta}^{-i}(i \leq M)$ such that $f_{\eta}^{-i}(p) \in \partial \Omega$. (Recall that $U$ is the neighborhood of $\partial \Omega$ where $f$ is defined.) The constant $M$ will be fixed later on (after the statement of the Main Lemma). Take $\beta \in(\alpha, \pi)$ and $r$ as in Lemma 2.

Since the radial limit of $R$ along $r_{\tau}$ exists, there is $r_{2} \in(0,1)$ such that $R(x) \in \frac{1}{2} B$ for $|x|>r_{2}, x \in r_{\tau}$. One can assume that $r_{2}>r$.

Let $y \in r_{2} \cdot \tau$. Take $x \in r_{\tau},|x|>r_{2}$, and $z \in T_{\alpha}(x)$ (Figure 2). Join the points $x$ and $z$ with the segment $\gamma$ of a straight line. (Obviously, $\gamma \subset T_{\alpha}(x)$.)

We claim that $R(\gamma) \subset B$ if $|x|$ is close to 1 .

Suppose the contrary, i.e. that $R(\gamma)$ crosses $\partial B$. Take a parametrization of $\gamma$ :

$$
[0,1] \ni t \mapsto \gamma(t)=t \cdot z+(1-t) \cdot x \text {. }
$$

Let $t_{0}=\sup \{t: \gamma([0, t]) \subset B\}$. Let $w=\gamma(t)$ for some $t \in\left(0, t_{0}\right)$. We compare $\log \left|R^{\prime}(y)\right|$ and $\log \left|R^{\prime}(w)\right|$ (see Figure 3). To do this, we apply to both $y$ and $w$ an appropriate branch of $g^{-n}$.

Fix $\delta<0, K>0$. Let $g_{\eta}^{-n}$ be a branch of $g^{-n}$ defined in $T_{\alpha}(x)$. This branch is called good if there exists a holomorphic branch $f_{\eta}^{-n}$ defined in $2 \cdot B$ and such that

$$
f^{-n}(p)=R\left(g_{\eta}^{-n}(\tau)\right)
$$




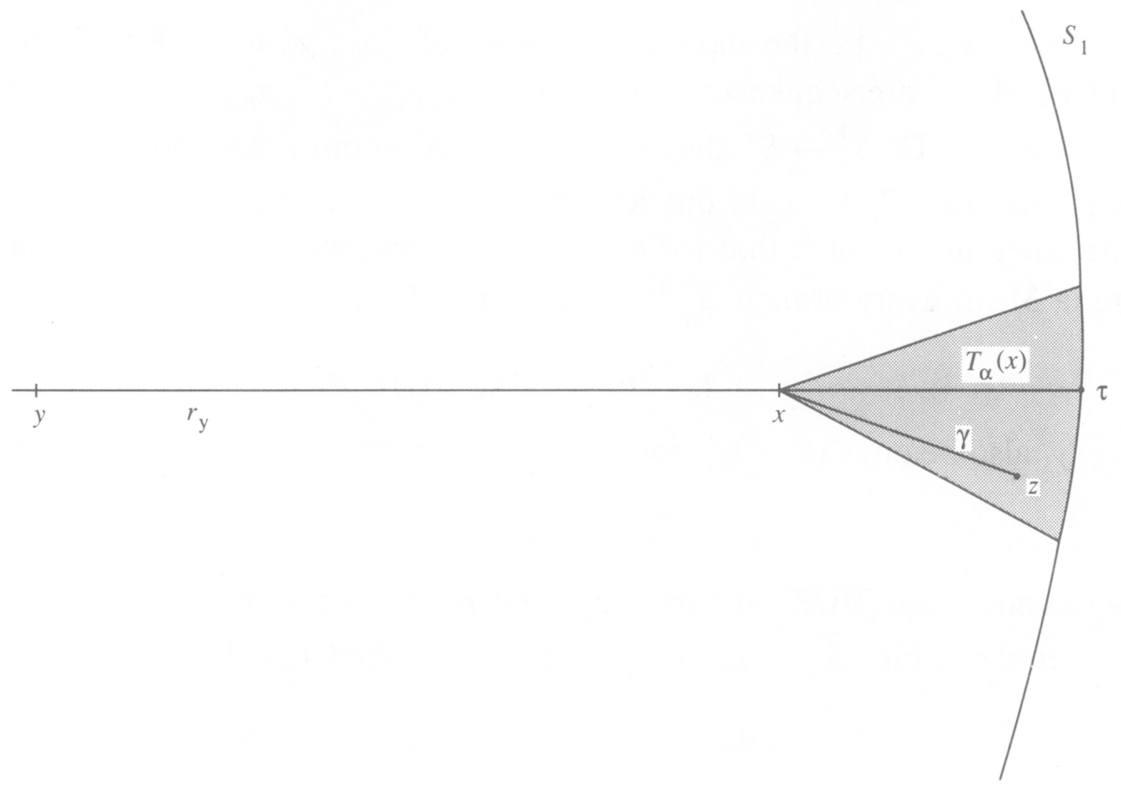

FIGURE 3

and

$$
\operatorname{diam}\left(f^{-n}(B)\right)<K \exp (-n \delta)
$$

We have

$$
R\left(g^{-n}(w)\right)=f_{\eta}^{-n}(R(w))
$$

and

$$
R\left(g_{\eta}^{-n}(y)\right)=f_{\eta}^{-n}(R(y))
$$

because $R(\gamma([0, t])) \subset B$ and $R\left(r_{\tau} \cap\left\{z:|z| \geq r_{2}\right\}\right) \subset B$. Thus, for a good branch $g_{\eta}^{-n}$, we have

$$
\begin{aligned}
\log \left|R^{\prime}(y)\right|-\log \left|R^{\prime}(w)\right|= & \left(\log \left|R^{\prime}\left(g_{\eta}^{-n}(y)\right)\right|-\log \left|R^{\prime}\left(g_{\eta}^{-n}(w)\right)\right|\right) \\
& -\log \left|\frac{\left(f_{\eta}^{-n}\right)^{\prime}(R(y))}{\left(f_{\eta}^{-n}\right)^{\prime}(R(w))}\right|+\log \left|\frac{\left(g_{\eta}^{-n}\right)^{\prime}(y)}{\left(g_{\eta}^{-n}\right)^{\prime}(w)}\right| .
\end{aligned}
$$

The third summand is bounded independently of $n$ (Lemma 2). If $g^{-n}$ is a good branch, then also the second summand is bounded, since $f^{-n}$ is defined in $2 \cdot B$ and $R(y), R(w)$ are in $B$ (use the Distortion Theorem for univalent functions, [Go]).

We are going to find for infinitely many $n$ good branches $g_{\eta}^{-n}$ such that $\log \left|R^{\prime}\left(g_{\eta}^{-n}(y)\right)\right|$ and $\log \left|R^{\prime}\left(g_{\eta}^{-n}(w)\right)\right|$ are bounded (independently of $n$ ).

The proof uses the construction made in [PUZ, Lemma 8, §5]. This lemma contains an explicit construction of the Pesin set. We shall formulate it here in a more convenient form. 
Let $\left(\widetilde{S}^{1}, \tilde{g}, \tilde{\nu}\right)$ be the natural extension of $\left(S^{1}, g, \nu\right)$. (Recall that the elements of $\tilde{S}^{1}$ are sequences $x=\left(\cdots x_{-k}, x_{-k+1}, \ldots, x_{0}, x_{1}, \ldots\right)$ such that $g\left(x_{i}\right)=x_{i+1}$.) $\Pi: \widetilde{S}^{1} \rightarrow S^{1}$ denotes the projection onto 0th coordinate.

The domain $T_{\alpha}(x)$ cuts out an $\operatorname{arc} L \subset S^{1}$. There is an arc $L_{w} \subset L$ (depending on $w$ ) such that for every $t \in L_{w}$ we have: $w \in S_{\alpha}(t)$. Then (by Lemma 3) for every branch $g_{\eta}^{-n}$ defined in $T_{\alpha}(x)$,

$$
g_{\eta}^{-n}(w) \in S_{\beta}\left(g_{\eta}^{-n}(t)\right) \text {. }
$$

Since $y$ also belongs to $S_{\alpha}(t)$ for $t \in L_{w}$, we have

$$
g^{-n}(y) \in S_{\beta}\left(g_{\eta}^{-n}(t)\right) \text {. }
$$

Main Lemma (see [PUZ, Lemma, §5]). There exist constants $\delta>0, M \in \mathbb{Z}_{+}$, $K>0$ and a subset $\widetilde{K}_{w} \subset \Pi^{-1}\left(L_{w}\right), \tilde{\nu}\left(\widetilde{K}_{w}\right)>0$ such that if

$$
q=\left(\ldots, q_{-k}, q_{-k+1}, \ldots, q_{0}, q_{1}, \ldots\right) \in \widetilde{K}_{w},
$$

then

$$
q_{-k}=g_{\eta}^{-k}\left(q_{0}\right) \text { for some good branch } g_{\eta}^{-k} \text {. }
$$

The following reasoning contains the ideas of [Li] (see also [PUZ, Lemma 1 $\S 1]$ ), which are used to solve problems of this kind for expanding maps.

Surprisingly, by a careful choice of good backward trajectories one obtains estimates very similar to those for expanding maps.

Define the function $v \in L^{2}\left(S^{1}, \nu\right)$ as

$$
v(x)=\lim _{\substack{z \rightarrow x \\ \text { nontan }}} \log \left|R^{\prime}(z)\right|
$$

wherever the limit exists. Since the function $v$ is $\nu$-measurable, one can find a subset $F \subset S^{1}$ with $\nu(F)>\frac{1}{2}$ and $r_{3}>0$ such that

$$
v_{\mid F} \text { is continuous, }
$$

$s \in S_{\beta}(q),|q-s|<r_{3}$ then

$$
\left|\log R^{\prime}(s)-v(q)\right|<K .
$$

Since the measure $\tilde{\nu}$ is ergodic, for $\tilde{\nu}$-almost all $\tilde{q} \in \widetilde{K}_{w} \quad \tilde{g}^{-n}(\tilde{q})$ falls into $\widetilde{F}=\Pi^{-1}(F)$ with a frequency $\tilde{\nu}(F)>\frac{1}{2}$. Take such a typical point $q=\left(q_{i}\right)_{i=-\infty}^{\infty} \in \widetilde{K}_{w}$. For $n$ such that $\tilde{g}^{-n}(\tilde{q}) \in \widetilde{F}$, we have

$$
\left|v\left(q_{-n}\right)\right|<K \quad(\text { by }(14)) \text {. }
$$

As we noticed above,

$$
g_{\eta}^{-n}(w) \in S_{\beta}\left(q_{-n}\right) \text { and } g_{\eta}^{-n}(y) \in S_{\beta}\left(q_{-n}\right),
$$


where $q_{-n}=g_{\eta}^{-n}\left(q_{0}\right)$. Obviously, for $n$ large

$$
\left|g_{\eta}^{-n}(w)-q_{-n}\right|<r_{3} \text { and }\left|g_{\eta}^{-n}(y)-q_{-n}\right|<r_{3} .
$$

Thus, we have

$$
\log \left|R^{\prime}\left(g_{\eta}^{-n}(w)\right)\right|<2 K \quad \text { and } \quad \log \left|R^{\prime}\left(g_{\eta}^{-n}(y)\right)\right|<2 K .
$$

This estimate, together with $(8)$, gives

$$
|\log | R^{\prime}(y)|-\log | R^{\prime}(w)||<K_{1}
$$

for some $K_{1}$ independent of $w \in \gamma$ and $x$, or, equivalently,

$$
\left|R^{\prime}(w)\right|<\left|R^{\prime}(y)\right| \cdot \exp K_{1} .
$$

This estimate shows that $R(\gamma)$ cannot cross $\partial B$ if $|x|$ is close to 1 . Indeed, notice that (18) is valid for every $w=\gamma(t), t \in\left[0, t_{0}\right]$. Thus,

$$
\text { length }\left(R\left(\gamma\left(\left[0, t_{0}\right]\right)\right)\right) \leq \text { length } \gamma\left(\left[0, t_{0}\right]\right) \cdot\left|R^{\prime}(y)\right| \cdot \exp K_{1} \underset{|x| \rightarrow 1}{\longrightarrow} 0 .
$$

Hence, if $|x|$ is close to $1, R\left(\gamma\left(\left[0, t_{0}\right]\right)\right)$ is too short to have the endpoint in $\partial B$.

Thus, $R\left(T_{\alpha}(x)\right) \subset B$. In this way, (a) is proved.

The statement of $(b)$ is a straightforward consequence of the proof of $(a)$ because, once (a) is proved, we can take as $w$ an arbitrary point of $T_{\alpha}(x)$. Proposition 2 is proved.

Proposition 3. For $\alpha, x$ as in the statement of Proposition 2 the function $T_{\alpha}(x) \ni$ $w \mapsto \log \left|R^{\prime}(w)\right|$ is uniformly continuous in $T_{\alpha}(x)$.

Proof is a modification of the proof of Proposition 2. Recall formula (8):

$$
\begin{aligned}
\log \left|R^{\prime}(y)\right|-\log \left|R^{\prime}(w)\right|= & \left(\log \left|R^{\prime}\left(g_{\eta}^{-n}(y)\right)\right|-\log \left|R^{\prime}\left(g_{\eta}^{-n}(w)\right)\right|\right) \\
& -\log \left|\frac{\left(f_{\eta}^{-n}\right)^{\prime}(R(y))}{\left(f_{\eta}^{-n}\right)^{\prime}(R(w))}\right|+\log \left|\frac{\left(g_{\eta}^{-n}\right)^{\prime}(y)}{\left(g_{\eta}^{-n}\right)^{\prime}(w)}\right| .
\end{aligned}
$$

Assume that both $y$ and $w$ are points of $T_{\alpha}(x), g_{\eta}^{-n}$ is, as before, a good branch. The second summand can be estimated by $C \cdot|R(x)-R(y)|$, where $C$ is some constant (by the Distortion Theorem, see [Go]). But we already know (Proposition 2, (b)) that $R_{\mid T_{\alpha}(x)}$ is uniformly continuous. Thus, the second summand tends to zero as $|x-y|$ tends to zero. that

To estimate the first summand we have to find a common branch $g_{\eta}^{-n}$ such

$$
g_{\eta}^{-n}(y) \in S_{\beta}\left(q_{y}\right) \text { for some } q_{y} \in F
$$

and

$$
g_{\eta}^{-n}(w) \in S_{\beta}\left(q_{w}\right) \text { for some } q_{w} \in F
$$

( $\beta$ is as in the statement of Lemma 3; see the proof of Proposition 2). 
Take the set $\widetilde{K}_{x}$ as in the statement of the Main Lemma. Let $\mathscr{P}$ be a partition of $\widetilde{K}_{x}$ given by a partition of $L_{x}$ into points. By $\tilde{\nu}_{q}$ we denote a conditional measure on the fiber $\mathscr{F}_{q}=\left\{\left(\ldots q_{-k}, q_{-k+1}, \ldots, q_{0}, q_{1}, \ldots\right): q_{0}=\right.$ $q\}$. Let $G=\left\{\tilde{q} \in \widetilde{K}_{x}: \tilde{g}^{-n}(\tilde{q})\right.$ fall into $F$ with a frequency $\left.>\frac{1}{2}\right\}$. The set $G$ has a full measure since $g$ is ergodic. Thus (by the definition of a conditional measure)

$$
\tilde{\nu}_{q}\left(G \cap \mathscr{F}_{x}\right)=1 \text { for a.e. } q \in L_{x} .
$$

Notice that we have a natural isomorphism between fibers of the partition $\Pi$ : the element $\left(\ldots q_{-k}, q_{-k+1}, \ldots, q_{0}, q_{1}, \ldots\right) \in \mathscr{F}_{q_{0}}$ is identified with $\left(\ldots p_{-k}, p_{-k+1}, \ldots, p_{0}, p_{1}, \ldots\right) \in \mathscr{F}_{p_{0}}$ iff $P_{-n}=g_{\eta}^{-n}\left(p_{0}\right)$ and $q_{-n}=g_{\eta}^{-n}\left(q_{0}\right)$ for some branch $g_{\eta}^{-n}$ defined in the neighborhood of $L$. Denote this identification by $\varphi_{p, q}: \Pi^{-1}\left(p_{0}\right) \rightarrow \Pi^{-1}\left(q_{0}\right)$. Notice that $\varphi_{p_{0}, q_{0}}$ is absolutely continuous with respect to $\tilde{\nu}_{p_{0}}, \tilde{\nu}_{q_{0}}$. It is so, because for every branch $g_{\eta}^{-n}$ defined in the neighborhood of $L$

$$
\tilde{\nu}_{p_{0}}\left(\left\{\tilde{r}: \tilde{r} \in \mathscr{F}_{p_{0}} \text { and } r_{-n}=g_{\eta}^{-n}\left(p_{0}\right)\right\}\right)
$$

is proportional to $\left|\left(g_{\eta}^{-n}\right)^{\prime}\left(p_{0}\right)\right|$, similarly for $q$. The corresponding ratio (proportional to $\left.\frac{\left|\left(g_{\eta}^{-n}\right)^{\prime}\left(p_{0}\right)\right|}{\left|\left(g_{\eta}^{-n}\right)^{\prime}\left(q_{0}\right)\right|}\right)$ is bounded by the distortion property.

Thus, for almost every $p \in L_{x}$ and $q \in L_{x}$

$$
\tilde{\nu}_{q}\left(\varphi_{p, q}\left(G \cap \mathscr{F}_{p}\right) \cap\left(G \cap \mathscr{F}_{q}\right)\right)=1 .
$$

We choose $p \in L_{w}$ and $q \in L_{y}$ satisfying (21). If $\tilde{r} \in \mathscr{F}_{q} \cap \varphi_{p, q}\left(G \cap \mathscr{F}_{p}\right)$, then with a frequency $\tilde{\nu}(\widetilde{F})>\frac{1}{2}$ we have $\tilde{g}^{-n}(\tilde{r}) \in \widetilde{F}$, and also with a frequency $\tilde{\nu}(\widetilde{F}), \tilde{g}^{-n}\left(\varphi_{p, q}^{-1}(\tilde{r})\right) \in \widetilde{F}$. Thus, one can find infinitely many $n$ 's so that

$$
\begin{aligned}
& g_{\eta}^{-n}(p) \in F, g_{\eta}^{-n}(q) \in F \text { for some } g_{\eta}^{-n} \text { defined in the neigh- } \\
& \text { borhood of } L .
\end{aligned}
$$

By the choice of $L_{w}, L_{y}$, and Lemma 3 we get

$$
g_{\eta}^{-n}(y) \in S_{\beta}\left(g_{\eta}^{-n}(p)\right) \text { and } g_{\eta}^{-n}(w) \in S_{\beta}\left(g_{\eta}^{-n}(q)\right) .
$$

This allows us to finish the proof (using the properties (13) and (15)). Proposition 3 is proved.

Corollary 1. (a) The derivative of the Riemann map $R$ is bounded in $P_{p_{0}, 1}$, and therefore $R$ is uniformly continuous and extends to a continuous map defined in $\bar{D}$. (The extended map will be still denoted by $R$.)

(b) The function $P_{p_{0}, 1} \ni w \rightarrow R^{\prime}(w)$ is uniformly continuous. Denote (as before) by $v$ the boundary value of $\log \left|R^{\prime}\right|$. Then

$$
v \circ g-v=\log \left|f^{\prime} \circ R\right|-\log \left|g^{\prime}\right| \text { on } S^{1} \text {. }
$$


(c) If $R(q) \quad\left(q \in S^{1}\right)$ is not a critical value, then $v$ is bounded in the neighborhood of $q$ in $S^{1}$.

Proof. (a) Take an arc $L^{\prime}$ such that $\operatorname{cl} L^{\prime} \subset T_{\alpha}(x) \cap S^{1}$. For some $k \in \mathbb{Z}_{+}$, $g^{k}\left(L^{\prime}\right)=S^{1}$. Since $\left(f^{k}\right)^{\prime} \circ R \cdot R^{\prime}=R^{\prime} \circ g^{k} \cdot\left(g^{k}\right)^{\prime}$ and $R_{\mid T_{\alpha}(x)}^{\prime}$ is bounded, $R^{\prime}$ is bounded in some annulus $r<|z|<1$.

(b) is proved in the same way; (24) is a consequence of a limit passage.

(c) follows from the proof of Proposition 2.

Corollary 1 ends the proof of Theorem 1.

Corollary 2. The map $R_{\mid S^{1}}$ is differentiable and the derivative $\frac{d}{d \vartheta}\left(R\left(e^{i \vartheta}\right)\right)$ is continuous. Moreover, if $R\left(e^{i \vartheta_{0}}\right)$ is not a critical value, then $\left|\frac{d}{d \vartheta} R\left(e^{i \vartheta}\right)\right| \vartheta_{0} \mid>$ 0 .

Corollary 3. (a) If $p \in \partial \Omega$ is not a critical value, then $p$ has finitely many preimages under $R$.

(b) If $q \in \partial \Omega$ is not a periodic point, then $q$ has finitely many preimages under $R$.

Proof. (a) follows immediately from Corollary 2 . To prove (b), take $k$ such that every $y$ satisfying $f^{k}(y)=q$ is not a critical value. Then every $y$ as above has only finitely many preimages under $R$ (by (a)) and the same must be true for $q$.

\section{CRitical points in $\partial \boldsymbol{\Omega}$}

Proposition 4. (a) If $c=R(q)$ is a critical point and $g^{k}(q)=g(s)$ for some $s \in S^{1}$, then $x=R(s)$ is a critical point or a critical value.

(b) If $c \in \partial \Omega$ is a critical point of $f$, then the trajectory of $c$ falls into a periodic orbit. This orbit is repelling.

Proof. (a) Denote by $p$ the point $f^{k}(c)$. Suppose that $x$ is neither a critical point nor a critical value. One can assume that $c$ is not a critical value. We already know that $\log \left|R^{\prime}\right|$ is bounded in neighborhoods of $s$ and $q$. The formula $\left(f^{k}\right)^{\prime} \circ R \cdot R^{\prime}=R^{\prime} \circ g^{k} \cdot\left(g^{k}\right)^{\prime}$ shows that in the neighborhood of $g^{k}(q)$, $\log \left|R^{\prime}\right|$ is unbounded (since $q \stackrel{g^{k}}{\longrightarrow} g^{k}(q) \stackrel{R}{\longrightarrow} p$ ); on the other hand it must be bounded (since $s \stackrel{g}{\longrightarrow} g^{k}(q) \stackrel{R}{\longrightarrow} p$ ). This contradiction proves (a).

(b) Assume that $c$ is not a critical value, $c=R(q), q \in S^{1}$. Take $k \in \mathbb{Z}_{+}$ such that for $l \geq k f^{l}(c)$ is not a critical point.

Now, take $j \in \mathbb{Z}_{+}$for which there exists $s \in S^{1}$ such that

$$
s \neq g^{k}(q), \quad g^{j}(s)=g^{k+j}(s)
$$

and

$$
R(s) \neq f^{k}(c)
$$


(The number $j$ satisfying (1) and (2) exists because otherwise all points $s$ satisfying (1) would have the same image $f^{k}(c)$ under $R$. This contradicts the continuity of $R$.) For some $i \leq j$ we have

$$
R\left(g^{i}(s)\right) \neq f^{k+i}(c)
$$

and

$$
R\left(g^{i+1}(s)\right)=f^{k+i+1}(c) .
$$

Denote $R\left(g^{i}(s)\right)$ by $x$. It follows from (a) that $x$ is either a critical point or a critical value.

Now, we repeat the whole procedure with $f^{k}(c)$ replaced by $f^{k+j}(c)$ and proceed by induction. Since there are finitely many critical points, one gets

$$
\begin{array}{ll}
f^{l}(c)=f^{m}(d), & f^{m-1}(d) \neq f^{l-1}(c), \\
f^{r}(c)=f^{n}(d), & f^{n-1}(d) \neq f^{r-1}(c),
\end{array}
$$

for some critical point $d \in \partial \Omega$ and $l, r, m, n \in \mathbb{Z}_{+}$or $f^{k}(c)$ is periodic. But (5) and (6) also imply that either $f^{l}(c)$ or $f^{r}(c)$ is periodic. We denote this point by $p$. Let $p=f^{l}(c)$, then $p=R\left(g^{l}(q)\right)$. We claim that the trajectory of $q$ is also finite.

One can assume that $f(p)=p$. As before, one can find $s=g^{i}(s) \in S^{1}$ such that

$$
x=R(s) \neq f^{l+i}(c)=p
$$

and

$$
R\left(g^{i+1}(s)\right)=f^{l+1}(c)=f(p)=p .
$$

Now, $x=R(s)$ is a critical value (by (a)) and $x$ is not periodic. By Corollary 3(b) $x$ has finitely many preimages under $R$. But also $x$ belongs to the finite set of critical values. Thus, there are only finitely many points $s$ which can satisfy (7).

Since this construction can be repeated infinitely many times, we get for some $s$ as above:

$$
g^{j_{1}}(q)=q^{r_{1}}(s)
$$

and

$$
g^{j_{1}}(q)=g^{r_{2}}(s)
$$

for some $j_{1}, j_{2}, r_{1}, r_{2} \in \mathbb{Z}_{+}$. This implies that $g^{j_{1}}(q)$ or $g^{j_{2}}(q)$ is periodic.

Finally, we check that $p$ is a source. One can assume that $p=R\left(g^{l}(q)\right)$, $g\left(g^{l}(q)\right)=g^{l}(q)$, and $f(p)=p$. It is easy to see that for $w \in D, w$ close to $r$,

$$
\log \left|R^{\prime}(w)\right| \approx\left(\operatorname{deg}_{c} f^{\prime}-1\right) \log \left|w-g^{l}(q)\right|
$$


where the sign $\approx$ means that the difference is bounded. Obviously,

$$
\log \left|R^{\prime}(g(w))\right|+\log \left|g^{\prime}(w)\right|=\log \left|f^{\prime}(R(w))\right|+\log \left|R^{\prime}(w)\right| .
$$

Putting (11) to the formula (12) and comparing it again with (11) (for $g(w)$ ), we get

$$
\log \left|f^{\prime}(p)\right|=\operatorname{deg}_{c} f^{l} \cdot \log \left|g^{\prime}\left(g^{l}(q)\right)\right| .
$$

(We omit a straightforward computation.)

Thus, $p$ is a source.

\section{5. $\partial \Omega$ IS ANALYTIC}

Proposition 5. If $\log \left|f^{\prime} \circ R\right|-\log \left|g^{\prime}\right|$ is homologous to zero and

(a) $p$ is an arbitrary point and there are no critical points in $\partial \Omega$, or

(b) $p$ is a critical point not being a critical value, then in a neighborhood of $p \quad \partial \Omega$ is a smooth arc.

Proof. Let $V$ be a small neighborhood of $p$. Then $R^{-1}(V \cap \partial \Omega)$ is a union of countably many arcs $l_{i} \subset S^{1}$. Since the derivative $\frac{d R}{d \vartheta}$ is uniformly bounded in $l_{i}$ 's (see Corollary $1, \S 3$ ), there is a neighborhood $U \subset V$ such that $U \cap L_{i} \neq \varnothing$ only for a finite number of curves $L_{i}=R\left(l_{i}\right)$ and $L_{i}$ have no self-intersections. Taking a smaller neighborhood one can assume that $p \in L_{i}$ for all the curves $L_{i}$ intersecting $U$.

But every $L_{i}$ is an image of an arc under a map with nonzero derivative, see Corollary $2, \S 3$. Thus, there are at most two arcs $L_{1}, L_{2}$, tangent at $p$.

First, consider (a). In this case $f_{\mid \partial \Omega}^{n}$ is expanding for some $n$ (because $\log \left|R^{\prime}\right|$ extends to a bounded function on $\bar{D}$, using Corollary $1, \S 3$ ). Now, the proof can be deduced directly from Lemma 2, $\S 4$ of [PUZ]. This lemma says that if $\log \left|f^{\prime} \circ R\right|$ is homologous to $\log \left|g^{\prime}\right|$ and $f_{\mid \partial \Omega}$ is expanding, then $\partial \Omega$ is a Jordan curve. The corresponding proof, however, is very complicated (it does not use the fact that $\partial \Omega$ is smooth). In the improved version of [PUZ], it will be replaced by the reasoning below.

We use two other (very simple) lemmas of [PUZ]. First, using Lemma 3, $\S 4$ [PUZ] we notice $f_{\mid \partial \Omega}$ expanding implies $\operatorname{cl} \Omega \neq \overline{\mathbb{C}}$. Then, having assumed that $\partial \Omega$ is not a Jordan curve, one can find a constant $K>0$ such that for every $r>0$ there exists $x \in \overline{\mathbb{C}}-\operatorname{cl} \Omega$ such that $\operatorname{dist}(p, x)<r$ and

$$
\operatorname{dist}(x, \partial \Omega) / \operatorname{dist}(x, p)>K \text {. }
$$

((1) is obvious for $r$ large (since $\mathrm{cl} \Omega \neq \overline{\mathbb{C}}$ ); take a ball $B(p, r)$ and $n$ so big that $f^{n}(B(p, r))$ is large; then go back. Using the distortion property we can keep the same constant $K$ for all $r$; see the proof of Sublemma of Lemma 2, $\S 4$ in [PUZ].)

But we already know that in the neighborhood of $p \partial \Omega$ is a sum of two smooth arcs, tangent at $p$. This contradicts (1) and proves (a). 
(b) In this case it is easy to see that $f\left(L_{1}\right)=f\left(L_{2}\right)$. Otherwise, one of these curves would intersect $\Omega$. Notice also that $\operatorname{deg}_{p} f=2$. Otherwise the point $f(p)$ would be in $\Omega$.

Now, $f\left(L_{1}\right) \quad\left(=f\left(L_{2}\right)\right)$ is either a smooth open-closed interval having $f(p)$ as its endpoint or a union of two such intervals, tangent at $f(p)$.

We know (see Proposition 4(b)) that for some $k>0, f^{k}(p)$ is a source, say $f\left(f^{k}(p)\right)=f^{k}(p)$ and $f^{\prime}\left(f^{k}(p)\right)=\alpha,|\alpha|>1$.

Moreover, $\operatorname{deg}_{f(p)} f^{k-1}=1$; otherwise $f^{k}(p)$ would be in $\Omega$.

Thus, in the neighborhood of $f^{k}(p), f^{k}(\partial \Omega \cap U)$ is again one smooth openclosed interval or a union of two such intervals $I_{1}, I_{2}$, tangent at $f^{k}(p)$.

The second possibility can be easily excluded by the following argument:

In the neighborhood of $f^{k}(p) f$ is smoothly conjugate to the multiplication by $\alpha$. It follows that $\alpha \in \mathbb{R}_{+}$, since otherwise $f\left(I_{1}\right), f\left(I_{2}\right)$ would intersect $\Omega$. But then for a small neighborhood $W$ of the point $f^{k}(p)$ we have

$$
\bigcup_{n=0}^{\infty} f^{n}(W \cap \Omega) \supset W .
$$

But $f^{n}(W \cap \Omega) \subset \Omega$. Thus, $f^{k}(p) \in \Omega$, a contradiction.

Hence, $f(\partial \Omega \cap U)$ is one interval and therefore $\partial \Omega \cap U$ consists of one arc (since $\left.\operatorname{deg}_{p} f=2\right)$. This ends the proof of $(b)$.

Corollary 4. If $\log \left|f^{\prime} \circ R\right|-\log \left|g^{\prime}\right|$ is homologous to zero then $\partial \Omega$ is topologically either

(a) a circle (if there are no critical points in $\partial \Omega$ ), or

(b) a closed interval (if there are critical points in $\partial \Omega$ ).

Proof. (a) is an immediate consequence of Proposition 5(a).

(b) Take a point $p \in \partial \Omega$ satisfying (b) of Proposition 5. In a small neighborhood of $p, \partial \Omega$ is a single arc $J$. For the small neighborhood $W$ of the point $f(p)$, we have

$$
f(J) \cap \Omega \supset W,
$$

where ( $p$ is a critical point) and $f(J)$ is an open-closed (topological) interval. Moreover, for $k \geq 1 f^{k}(J)$ has no self-intersections (otherwise some points of $f^{k} J$ would lie in $\Omega$ ) and $f^{k}(J)$ contains a neighborhood $f^{k} W$ of $f^{k}(J)$. Thus, in the neighborhood of $f^{k}(J)$ there are no other points of $\partial \Omega$. It follows that $f^{k}(J) \cup f^{l}(J) \quad(k, l \geq 0)$ is an arc if the intersection is nonempty. Therefore, the whole boundary $\partial \Omega=\bigcup_{k=0}^{N} f^{k}(J)$ (for some $N \geq 1$ ) is topologically a closed interval.

In the next proposition we finish the proof by showing that $\partial \Omega$ is analytic.

Proposition 6. If $\log \left|f^{\prime} \circ R\right|$ is homologous to $\log \left|g^{\prime}\right|$ then $\partial \Omega$ is either

(a) an analytic Jordan curve or

(b) an analytically embedded closed interval. 
Proof. If there are no critical points in $\partial \Omega$, then $f$ is expanding (see the proof of Proposition 5(a)). Now, one adapts the ideas of Sullivan [Su] to prove that $\partial \Omega$ is actually analytic (see [P3] or [PUZ, $\S 6$, Theorem C] for details).

If there are critical points in $\partial \Omega$, then $\partial \Omega$ is (topologically) an interval (Corollary 4(b)). One can assume that the endpoints of this interval are $1,-1$ and $f(1)=1$.

Consider the map $\Pi: \overline{\mathbb{C}} \rightarrow \overline{\mathbb{C}}, \Pi(z)=\frac{z+z^{-1}}{2}, \Pi$ is a two-sheet covering of $\overline{\mathbb{C}}$ ramified at the points $1,-1$ and $\gamma=\Pi^{-1}(\partial \Omega)$ is a Jordan curve. $\gamma$ dissects $\overline{\mathbb{C}}$ into two topological discs $D_{1}, D_{2}$, each of them being mapped by one-to-one onto $\overline{\mathbb{C}}-\partial \Omega$.

The map $f$ can be lifted to both $D_{1}$ and $D_{2}$ by a formula $\tilde{f}=\Pi^{-1} \circ f \circ \Pi$.

The lifted maps glue to a continuous map defined on the neighborhood of $\gamma$.

Now, both $D_{1}$ and $D_{2}$ are RB-domains for $\tilde{f}$. One can easily check that $\tilde{f}_{\mid \gamma}$ has no critical points. Moreover, if $\widetilde{R}$ is a Riemann map onto, say, $D_{1}$ and $\tilde{g}=\widetilde{R}^{-1} \circ \tilde{f} \circ \widetilde{R}$, then $\log \left|\tilde{f}^{\prime} \circ \widetilde{R}\right|$ is homologous to $\log \left|\tilde{g}^{\prime}\right|$. (Otherwise $\gamma$ would not be rectifiable; compare Theorem 2 in [Z].)

Using (a), we conclude that $\gamma$ is a real-analytic Jordan curve. Let $S^{1} \ni$ $t \rightarrow \gamma(t)$ be a parametrization; we know that it extends to a holomorphic map defined on a neighborhood of $S^{1}$ in $\overline{\mathbb{C}}$. Denote by $t_{-1}, t_{1}$ the points which are mapped to $-1,1$ respectively.

Then the composition $\left[t_{-1}, t_{1}\right] \ni t \rightarrow \Pi(\gamma(t))$ gives the required analytic parametrization defined on the arc $\left[t_{-1}, t_{1}\right] \subset S^{1}$.

\section{REFERENCES}

[B] R. Bowen, Equilibrium states and ergodic theory of Anosov diffeomorphisms, Lecture Notes in Math., vol. 470, Springer-Verlag, Berlin-Heidelberg-New York, 1975.

[D] P. L. Duren, Theory of $H^{p}$ spaces, Academic Press, New York and London, 1970.

[Go] G. M. Goluzin, Geometric theory of functions of a complex variable, Transl. Math. Monographs, vol. 26, Amer. Math. Soc., Providence, R.I., 1969.

[Ma] A. Manning, The dimension of the maximal measure for a polynomial map, Ann. of Math. 119 (1984), 425-430.

[Mk1] N. G. Makarov, Dominating subset, the support of harmonic measure and perturbations of the spectra of operators in Hilbert space, Dokl. Akad. Nauk SSSR 274 (1984), 1033-1037. (Russian)

[Mk2] _ On the distortion of boundary sets under conformal mappings, Proc. London Math. Soc. (3) 51 (1985), 369-384.

[Ø] B. Øksendal, Brownian motion and sets of harmonic measure zero, Pacific J. Math. 95 (1981), 179-192.

[P1] F. Przytycki, Hausdorff dimension of harmonic measure on the boundary of an attractive basin for a holomorphic map, Invent. Math. 80 (1985), 161-179.

[P2] _ Riemann map and holomorphic dynamics, Invent. Math. 85 (1986).

[P3] - On holomorphic perturbations of $z \rightarrow z^{n}$, Bull. Polish Acad. Sci. Math. 34 (1986).

[Ph-St] W. Phillipp and W. Stout, Almost sure invariant principles for partial sums of weakly dependent random variables, Mem. Amer. Math. Soc., no. 161, 1975. 
[PUZ] F. Przytycki, M. Urbański, and A. Zdunik, Harmonic, Gibbs and Hausdorff measures on repellers for holomorphic maps, preprint, Univ. Warwick, 1986; part I, Ann. of Math. 130 (1989), 1-40; part II, Studia Math. (to appear).

[Su] D. Sullivan, Seminar on conformal and hyperbolic geometry by D. P. Sullivan (Notes by M. Baker and J. Seade), preprint IHES, 1982.

[Z] A. Zdunik, Parabolic orbifolds and the dimension of the maximal measure for rational maps, Invent. Math. 99 (1990), 627-649.

Institute of Mathematics, Warsaw University, 00-913 Warszawa 59, ul. Banacha 2, POLAND 